
allemande

47-1| 2015

L'Allemagne et l'Europe | La déprise de l'Empire napoléonien en Allemagne en 1813

\title{
Au nom de l'Europe. Les fondations politiques allemandes face à l'intégration européenne
}

Dorota Dakowska

\section{OpenEdition}

\section{Journals}

Édition électronique

URL : https://journals.openedition.org/allemagne/453

DOI : 10.4000 /allemagne.453

ISSN : 2605-7913

Éditeur

Société d'études allemandes

Édition imprimée

Date de publication : 26 juin 2015

Pagination : $37-46$

ISSN : 0035-0974

Référence électronique

Dorota Dakowska, « Au nom de l'Europe. Les fondations politiques allemandes face à l'intégration européenne », Revue d'Allemagne et des pays de langue allemande [En ligne], 47-1 | 2015, mis en ligne le 13 décembre 2017, consulté le 19 mai 2021. URL : http://journals.openedition.org/allemagne/453 DOI : https://doi.org/10.4000/allemagne.453 


\section{Au nom de l'Europe. Les fondations politiques allemandes face à l'intégration européenne}

\section{- Dorota Dakowska*}

Dès leur émergence dans l'après-Seconde Guerre mondiale, les fondations politiques allemandes - proches des partis politiques représentés au Bundestag - ont placé l'intégration européenne au cœur de leurs préoccupations. Si une de leurs missions initiales fut de proposer des programmes de «formation politique et civique " (politische Bildung) et de promouvoir le pluralisme dans une société ouest-allemande en voie de démocratisation, les responsables de ces organisations ont rapidement saisi l'importance du projet communautaire.

La Fondation Friedrich Ebert, dont les racines remontent à la République de Weimar, fut la première à développer une activité internationale. Dès les années cinquante, la Fondation dispensa des bourses aux étudiants allemands et étrangers et anima des rencontres internationales. Tout en déployant son travail dans les pays en développement, notamment africains, elle organisa de nombreux séminaires, conférences et programmes de formation pour des fonctionnaires, des syndicalistes et des journalistes $^{(1)}$. Les dirigeants de la Fondation rencontrèrent régulièrement des responsables de la Communauté économique européenne (CEE), des offices des Nations unies et d'autres institutions internationales. Cette familiarité avec les forums internationaux constitua une ressource pour la Fondation qui plaça au cœur de son discours la promotion de «l'entente entre les peuples » et des organisations multilatérales. Selon cette interprétation, la bonne connaissance, par les citoyens, des structures internationales renforcerait la légitimité de ces dernières et contribuerait à les pérenniser. En même temps, ces activités à destination du public valurent à la Fondation la reconnaissance

\footnotetext{
* Maître de conférences en science politique à Sciences Po Strasbourg, Université de Strasbourg, SAGE (UMR 7363).

1 Archiv der sozialen Demokratie (AdsD), fonds DW8 - 6c1, Die Welt, 16.01.1959.
} 
par ces institutions, elles-mêmes en quête de légitimité(2). Cette reconnaissance internationale fut utile sur le plan intérieur, pour confirmer l'expertise de la Fondation en matière de la coopération internationale et de l'aide au développement.

Cet article s'appuie sur nos recherches portant sur la genèse et l'institutionnalisation des fondations politiques allemandes et de leurs homologues européennes, entamées dans le cadre de notre thèse de science politique et développées depuis ${ }^{(3)}$. Dans un premier temps, le dispositif des fondations sera replacé dans son contexte institutionnel. Dans la deuxième partie, la place des fondations dans la politique étrangère allemande et dans les réseaux transnationaux de partis sera abordée avec une attention particulière portée à leur implication dans les changements de régime, notamment en Europe. La troisième partie est consacrée à l'investissement de l'arène bruxelloise par les fondations et à leur implication croissante dans des structures multilatérales. Enfin, dans la dernière partie, les stratégies de soutien aux élites pro-européennes sont discutées, en tant qu'elles ont constitué le cœur de l'investissement politique des fondations aussi bien en Europe du Sud que dans le Centre-Est européen. Si le soutien à l'intégration européenne et à l'adhésion de nouveaux États membres à l'Union européenne pouvait être considéré, de l'extérieur, comme une cause relativement consensuelle, cet article met en lumière les tensions, voire les paradoxes que cet engagement peut aussi impliquer.

\section{Situer les fondations politiques dans le champ politique allemand}

À la fois discrètes et incontournables sur la scène de l'« assistance internationale à la démocratie ", les fondations politiques sont des acteurs peu connus de la politique étrangère allemande. Alors qu'elles sont financées quasi entièrement sur fonds publics, elles disposent d'une considérable autonomie d'action. Indissociables des partis politiques, elles ne peuvent pas pour autant être réduites à des instruments de ces derniers. De fait, c'est grâce à leur ancrage simultané dans deux champs d'action - le champ politique et celui de l'action publique extérieure - que les fondations parviennent à accumuler des ressources conséquentes, tant en termes financiers que symboliques, de capital politique et de légitimité.

La croissance constante de leurs subventions s'explique à la lumière de leurs liens avec les partis politiques. Les six fondations politiques officiellement reconnues à ce jour sont en effet proches des principaux partis représentés au Bundestag ${ }^{(4)}$.

2 AdsD, DW8 - 6c1, Wie Feder, 30 septembre 1964; "Coopération FES - ONU ", 19.06.1959, ppp, 08.06.1959.

3 Dans ce cadre, plus de 140 entretiens ont été conduits dans les années 2000-2004 puis 2007-2014 à Berlin, Bonn, Munich, Bruxelles, Budapest, Varsovie, Paris et Amsterdam avec les responsables des fondations dans leurs sièges en Allemagne et leurs bureaux à l'étranger, auprès de leurs ministères de tutelle, des partis dont elles sont proches, mais aussi avec leurs principaux partenaires. Ces données ont été complétées par le dépouillement de plusieurs fonds d'archives, en particulier les archives de fondations ainsi que ceux du ministère fédéral des Affaires étrangères (Auswärtiges Amt).

4 L'attribution des subventions prend en compte les résultats des dernières élections législatives. Elle n'est pas automatique et dépend d'un accord politique entre les principaux groupes parlementaires. Les Verts, puis le PDS se sont mobilisés, respectivement dans les années 1980 et 1990, pour obtenir la reconnaissance de « leur» fondation. Cf. Dorota DA Kowska, Le pouvoir des fondations. Des acteurs de la politique étrangère allemande, Rennes, Presses universitaires de Rennes, 2014. 


\section{Les fondations politiques allemandes (politische Stiftungen)}

Friedrich-Ebert-Stiftung - proche du Parti social-démocrate (SPD), créée en 1925, interdite en 1933 et réactivée en 1947

Konrad-Adenauer-Stiftung - proche de l'Union chrétienne-démocrate (CDU), créée en 1964 à partir de deux instituts préexistant depuis 1955 et 1962

Friedrich-Naumann-Stiftung - proche du Parti libéral-démocrate (FDP), créée en 1958

Hanns-Seidel-Stiftung - proche de l'Union chrétienne-sociale (CSU), créée en 1967

Heinrich-Böll-Stiftung - proche des Verts (Bündnis 90/Die Grünen), créée en 1997 à partir de trois organisations financées depuis 1989

Rosa-Luxemburg-Stiftung - proche à l'origine du Parti du socialisme démocratique (PDS) et désormais du parti de Gauche (Die Linke), officiellement reconnue en 1999

Si leurs structures sont formellement indépendantes et si aucun transfert financier ne doit avoir lieu entre le parti et «sa » fondation, les liens entre ces entités demeurent nombreux. Historiquement proches des partis politiques - en tant que leurs émanations dans certains cas -, les fondations ont acquis progressivement une autonomie certaine, aidées par les décisions successives du Tribunal constitutionnel relatives au financement des partis politiques. Les liens entre les partis et les fondations se matérialisent à travers des responsables politiques, actifs ou en disponibilité - députés, ministres, ministres-présidents, voire chanceliers - qui siègent dans leurs conseils d'administration. Les directeurs généraux des fondations sont le plus souvent issus des instances dirigeantes de partis, tout en cumulant cette position avec d'autres fonctions électives ou non électives (ministre-président, haut responsable de l'administration, etc.). Ce capital politique accumulé sert les fondations de différentes manières. Il a permis l'augmentation constante de leurs budgets au cours des décennies. De plus, il accroît le rayonnement international des fondations puisque les responsables de leurs bureaux à l'étranger deviennent des intermédiaires capables de faciliter l'accès aux milieux du pouvoir en Allemagne.

Les activités internationales des fondations impliquent plusieurs centaines de délégués à l'étranger (sur deux mille employés au total), qui connaissent bien le pays dans lequel ils interviennent et en maîtrisent souvent la langue. Diplômés du supérieur (science politique, économie, droit notamment), ces responsables, qui choisissent d'effectuer tout ou une partie de leur carrière au sein de la fondation, disposent d'une expérience internationale et, souvent, de contacts solides dans les réseaux partisans allemands et transnationaux. Dans leur travail de terrain, ces représentants s'appuient sur le personnel local. Ils entretiennent un échange rodé avec les sièges des fondations en Allemagne et avec les ministères fédéraux qui sont leurs bailleurs de fonds pour le travail à l'étranger: le ministère des Affaires étrangères (AA) et le ministère de la Coopération économique et du Développement (BMZ).

\section{Accompagner les changements de régime, y compris en Europe}

Les répertoires d'action des fondations - dialogue inter-partisan et syndical, travail de formation politique, bourses, publications, conférences, voyages d'étude - s'appuient sur une expérience longue. Grâce aux fonds publics dont elles bénéficient (dont le montant total, plus de 460 millions d'euros annuels, dépasse aujourd'hui de loin 
l'ensemble des subventions publiques aux partis politiques allemands) et à une relative flexibilité de leur action, les fondations se sont impliquées dans les transformations politiques en Europe du Sud dans les années 1970, puis en Amérique latine dans les années 1980.

Si la contribution des fondations aux changements de régime demeure difficile à cerner, elle donne lieu, dans certains cas, à des récits héroïques, comme dans le cas du soutien apporté par la Fondation Friedrich Ebert à l'opposition portugaise précédant la «Révolution des œillets». Les porte-parole de la Fondation se plaisent à rappeler que le Parti socialiste portugais a été créé en 1973 dans un de ses centres de conférences, à Bad Münstereifel, où Mario Soares, alors en exil en France, fut accueilli pour organiser le congrès fondateur. Aussi bien au Portugal qu'en Espagne, la Fondation Ebert a apporté son soutien aux élites pro-européennes et non-communistes, aux militants politiques et syndicaux, en contribuant à créer des fondations proches de leurs mouvements. Si les fondations ont accompagné de nombreux changements de régime, c'est en Europe qu'elles ont pu jouer un rôle d'intermédiaires particulièrement recherchés, du fait de contacts dont elles disposaient au sein des familles politiques européennes.

Ces ressources ont pu être déployées en Europe du Centre-Est où les fondations s'étaient implantées dès 1989. Elles y ont développé des contacts avec les milieux politiques et académiques, contacts noués parfois bien avant la chute du mur de Berlin. L'Ostpolitik de Willy Brandt représentait en effet une opportunité pour la Fondation Friedrich Ebert sociale-démocrate qui proposa des échanges de journalistes, des programmes de bourses et un dialogue avec les élites communistes réformatrices ${ }^{(5)}$. La chute des régimes communistes a permis aux fondations de s'implanter plus durablement dans la région et d'ouvrir des bureaux de représentation dans ses principales capitales.

Cela étant dit, le travail des fondations ne repose pas seulement sur une mobilisation ponctuelle de moyens financiers pour soutenir des mouvements politiques et sociaux (à travers des formations, des voyages d'étude, etc.). Si de tels moyens d'action appartiennent à la panoplie assez classique des promoteurs de la démocratie, la force des fondations politiques allemandes s’appuie sur des liens tissés dans la durée. Ces liens s'inscrivent dans les réseaux européens de partis dont les fondations sont proches. La difficulté à cerner le travail des fondations est liée à leur inscription simultanée dans l'action publique extérieure et dans les réseaux partisans, allemands et transnationaux. Ces deux aspects se complètent davantage qu'ils ne s'excluent mutuellement. Cette double affiliation apparaît comme une ressource pour les fondations qui peuvent jouer, au besoin, sur l'un ou l'autre plan pour éviter une ingérence indésirable dans leurs projets.

Dans des contextes politiques soumis à des pressions internationales contradictoires et à des tensions politiques internes (régime autoritaire ou post-dictatorial), les fondations soutenaient les mouvements politiques via des organisations qui leur étaient proches et qu'elles ont, dans certains cas, contribué à créer. La coopération avec les

5 Dorota Dakowska, «Die Arbeit der Friedrich-Ebert-Stiftung in Polen zwischen 1971 und der friedlichen Revolution ", Archiv für Sozialgeschichte, vol. 45, 2005, dossier "West-Ost-Verständigung im Spannungsfeld von Gesellschaft und Staat seit den 1960er Jahren », p. 325-352. 
centres de recherche, les mouvements de jeunesse, les professionnels des médias revêt ainsi systématiquement une dimension politique, puisque ces organisations s'inscrivent dans un système d'action politiquement marqué. Si le soutien aux partis politiques et l'implication dans les campagnes électorales restent en principe interdits aux fondations, il serait illusoire de prétendre esquisser une démarcation univoque entre leurs activités de "formation sociopolitique » et un soutien à certains mouvements politiques.

À plusieurs reprises, les stratégies des fondations sont venues renforcer celles déployées par les internationales partisanes. Sans s'imposer de manière uniforme dans tous les contextes, cette proximité peut être expliquée par plusieurs facteurs. Une forte implication des leaders allemands dans les structures de ces réseaux partisans transnationaux peut contribuer à un rapprochement avec les fondations, comme ce fut le cas dans les années soixante-dix à l'époque de Willy Brandt, pour l'Internationale socialiste. Le soutien aux partis socialistes émergeants au Portugal et en Espagne fourni, dans les années 1970, par la Fondation Ebert en coopération avec l'Internationale socialiste s'inscrit dans ce contexte. De même, dans les années 1990 et à la veille de l'élargissement de l'Union européenne à l'Est, la familiarité des responsables de la Fondation Adenauer avec les instances dirigeantes de la CDU mais aussi avec les structures du Parti populaire européen (PPE) a facilité les échanges étroits avec les bureaux des fondations présentes dans les pays candidats ${ }^{(6)}$. Bien que le montant des subventions dont elles bénéficient ne puisse pas être traduit directement en influence politique, il est difficile de négliger le facteur matériel. Les structures partisanes transnationales (qu'il s'agisse d'internationales ou de fédérations européennes de partis) bénéficiant de moyens relativement modestes, le concours des bureaux des fondations allemandes constituait une ressource considérable.

Si leurs répertoires d'action se ressemblent dans les grandes lignes, les fondations diffèrent par leurs ressources, leur expérience, leurs priorités thématiques et politiques, en fonction du courant dont elles sont proches. En ce qui concerne leur rapport à l'intégration européenne, si les fondations plus anciennes, proches des partis établis et accédant régulièrement au gouvernement fédéral partagent une position de soutien relativement homogène, des variations sont à noter auprès des fondations plus récentes, la Fondation Heinrich Böll mais surtout la Fondation Rosa Luxemburg. Cette dernière représente la ligne la plus critique vis-à-vis de l'intégration européenne - conformément au discours du parti de Gauche (Die Linke) dont elle est proche. Cette ligne, qui passe par la critique des inégalités que les politiques économiques néo-libérales au sein de l'Union européenne pouvaient renforcer, peut varier en fonction du pays d'implantation. En Pologne, la Fondation Luxemburg a pu soutenir certaines personnalités issues des formations pro-européennes, telle l'Alliance de gauche démocratique (SLD), héritière de l'ancien Parti ouvrier unifié polonais mais relativement libérale sur le plan économique.

6 Dorota DakowskA, « German Political Foundations: Transnational party go-betweens in the process of EU enlargement ", in: Wolfram KaIser, Peter Starie (dir.), Transnational European Union, Londres, Routledge, 2005, p. 150-169. 


\section{Investir Bruxelles: des structures de plus en plus multilatérales}

L'investissement européen des fondations passe par l'ouverture des bureaux à Bruxelles, depuis la fin des années $1970^{(7)}$. Elles y entretiennent des contacts avec les institutions européennes: le Parlement européen et la Commission, en particulier en ce qui concerne le suivi de sa politique d'aide au développement. Plus récemment, dans les années 2000, les fondations allemandes présentes à Bruxelles ont contribué au développement de réseaux de fondations politiques au niveau européen.

Grâce à cet investissement, les fondations peuvent être qualifiées d'auxiliaires de l'espace politique européen ${ }^{(8)}$. Si elles facilitent l'accès aux institutions européennes à leur clientèle politique située en Allemagne ou à l'étranger, leur capacité à faire venir à Bruxelles des personnalités prometteuses des pays candidats, voisins ou partenaires de l'UE, nourrit l'attention que des responsables communautaires portent aux fondations. Dans cette perspective, les directeurs des bureaux bruxellois des fondations apparaissent comme des courtiers qui mettent en relation des professionnels de la politique bruxellois et étrangers ${ }^{(9)}$.

Si Bruxelles constitue un lieu d'implantation stratégique pour les fondations, celles-ci s'étaient néanmoins heurtées à des barrières en termes d'image et de reconnaissance dans l'espace communautaire. Identifiées avec un seul pays et dotées d'un profil politique assumé, les fondations cadraient mal avec l'attente de la Commission européenne qui privilégiait les contacts avec les groupes d'intérêt économiques ou civiques. Grâce à une mobilisation auprès de la Commission et du Parlement européen - appuyée par plusieurs eurodéputés influents - deux réseaux inédits ont vu le jour et gagné une reconnaissance à Bruxelles. Le premier d'entre eux est le Réseau européen de fondations politiques (European Network of Political Foundations, ENoP), créé en 2006, qui réunit près de 70 organisations issues de différents pays européens. Se positionnant dans le domaine de la politique d'aide au développement, ce réseau est parvenu à obtenir des financements de la Commission européenne. La deuxième initiative, ayant fait l'objet d'un règlement adopté en décembre 2007 (1524/2007) qui révise le statut des partis politiques au niveau européen défini en 2003 (règlement 2004/2003), institue des "fondations politiques au niveau européen ", à savoir des organisations transnationales et proches des « partis politiques au niveau européen ». Ces nouvelles structures se sont vu assigner la mission d'accompagner le travail des partis politiques au niveau européen à travers l'analyse et l'implication dans les débats sur l'intégration européenne, l'organisation de conférences et de formations et, enfin, en proposant un cadre permettant de réunir les fondations politiques nationales, des chercheurs et des acteurs politiques. Ces nouvelles fondations incluent des représentants des groupes politiques au Parlement européen, du « parti » transnational auquel

7 La FES fut la première à ouvrir son bureau à Bruxelles, en 1973. La KAS suivit en 1978, la FNS en 1985. La Fondation Böll ouvrit son bureau en 1998 et la Fondation Luxemburg en 2008.

8 Didier Georgakakis (dir.), Les métiers de l'Europe politique. Acteurs et professionnalisations de l'Union européenne, Strasbourg, Presses universitaires de Strasbourg (GSPE, sociologie politique européenne), 2002.

9 Sydney Tarrow, "La contestation transnationale ", Cultures et Conflits, n 38-39, 2000, dossier "Sociologie de l'Europe. Mobilisations, élites et configurations institutionnelles », p. 187-223, ici p. 214. 
il est lié et des fondations nationales qui leur sont affiliées. Conformément à la législation, les organes directeurs des fondations reflètent une composition " géographiquement équilibrée ». Cela étant, l'expertise et les ressources des membres allemands ont pesé dans la mise en place de ces organisations. Dans la plupart des cas, le premier secrétaire général nommé à la tête d'une nouvelle fondation fut le représentant d'une fondation allemande ${ }^{(10)}$.

Le modèle allemand des fondations politiques a-t-il réussi de cette manière sa percée définitive? S'il est parvenu à une reconnaissance institutionnelle prouvant qu'il est en partie reproductible, la réplication de ce modèle connaît cependant de nombreuses limites. Les différentes formes hybrides inspirées de la pratique allemande ne bénéficient ni des ressources, ni de l'autonomie, ni de la visibilité comparables ${ }^{(11)}$.

\section{Soutenir les élites pro-européennes: un enjeu consensuel?}

Depuis le début de leur engagement officiel en Europe centrale après la chute des régimes communistes, les représentants des fondations allemandes se sont employés à promouvoir, outre les thématiques spécifiques à chacune des fondations, le projet de rapprochement avec l'Union européenne, voire celui de l'adhésion.

Le financement des organisations formellement non partisanes permet de soutenir des militants et proches de partis politiques qui y sont employés, d'entretenir des groupes de sympathisants, d'encourager leur coopération, voire de fournir un soutien matériel (véhicules de fonction, matériel d'imprimerie, postes administratifs, etc.). En Pologne, la KAS a cherché à soutenir les élites libérales et pro-européennes, en finançant, dès les années 1990, la Fondation polonaise Robert Schuman, proche de l'Union de la liberté ou encore l'Institut de recherches sur l'économie de marché de Gdańsk, un think tank libéral proche des gouvernements du centre-droit ${ }^{(12)}$. Ce type d'investissements profite clairement aux deux parties: aux instituts en question, il fournissait des ressources et une visibilité supplémentaire, à la fondation, il octroyait des données de première main et des contacts précieux qui ajoutaient au prestige des manifestations qu'elle organisait et témoignaient de son insertion dans les réseaux d'expertise locale ${ }^{(13)}$. La Fondation Ebert a, pour sa part, organisé de nombreuses conférences

10 Ce fut le cas de la Fondation européenne d'études progressistes (FEPS) proche du Parti socialiste européen (PSE) mais aussi de la fondation proche des Verts (Green European Foundation). Le Centre for European Studies, proche du Parti populaire européen, fut rebaptisé, en mars 2014, Wilfried Martens Centre for European Studies pour honorer son président, ancien Premier ministre belge, président du PPE (1990-2013), décédé en 2013. Parmi les membres du conseil d'administration du Centre Martens, on retrouve Hans-Gert Pöttering, ancien président du Parlement européen, qui préside la Fondation Adenauer. Cf. Dorota Dakowska, «Vers une politisation du débat public européen? L'institutionnalisation des "fondations politiques européennes" ", Politique européenne, n 34, 2011, p. 167-199.

11 Certains passages de cette contribution sont basés sur notre article publié en ligne, Dorota DAкошsкA, "Les fondations politiques allemandes: des courtiers de la politique étrangère ", Ceriscope "Puissance ", novembre 2013, http://ceriscope.sciences-po.fr/puissance/content/part2/ les-fondations-politiques-allemandes-des-courtiers-de-la-politique-etrangere.

12 Le directeur de cet institut, l'économiste Janusz Lewandowski, fut ministre des Privatisations dans les années 1991 et 1992-1993, élu député européen en 2004, commissaire européen en charge de la Programmation financière et de budget entre 2009 et 2014.

13 Pour plus de précisions, cf. D. Dakowska, Le pouvoir des fondations (note 4). 
et formations et publié des ouvrages signés par des experts proches de l'Alliance de gauche démocratique, avec l'objectif d'accompagner le processus d'adhésion à l'Union européenne et de resserrer les liens entre le SPD et le SLD.

Cette activité s'inscrit dans les priorités des principales formations politiques en Allemagne, puisque les partis dont les grandes fondations sont proches poursuivent traditionnellement une ligne favorable à l'intégration européenne. Ce volet pouvait paraître relativement peu controversé dans la plupart des pays d'Europe centrale, dont les gouvernements avaient défini l'adhésion comme un objectif stratégique et qui ont rejoint l'UE dans l'une des trois vagues de l'élargissement vers l'Est. En même temps, la dimension politique de ce soutien est devenue de plus en plus explicite à mesure que les controverses autour de l'enjeu européen s'accentuèrent sur les scènes politiques nationales ${ }^{(14)}$. Le soutien aux formations résolument pro-européennes permit de renforcer l'environnement de ces partis, puisque ce sont les centres de recherche, les think tanks et les organisations de jeunesse proches de ces partis qui bénéficièrent prioritairement des financements allemands. Les leaders des mouvements plus conservateurs ou souverainistes pouvaient alors se sentir marginalisés.

Le soutien politique pré-électoral aux forces pro-européennes a pu avoir un retentissement politique particulier dans les cas où le paysage politique était fortement clivé autour de l'enjeu européen précisément. Le cas de soutien à l'opposition slovaque lors des élections de 1998, qui écartèrent du pouvoir Vladimir Mečiar, en apporte une illustration. Dans ce cas précis, les fondations allemandes se mobilisèrent pour promouvoir le changement de régime en Slovaquie. La Fondation Konrad Adenauer légitima Mikuláš Dzurinda, leader du Mouvement chrétien démocrate (KDH), et soutint la création de la Coalition démocratique slovaque (SDK). Aussi bien Dzurinda que le président slovaque Kovač furent invités à Bonn par la KAS pour y rencontrer des membres du groupe parlementaire CDU, dont la présidente du Bundestag. Le représentant de la KAS à Prague et à Bratislava consultait ses décisions non seulement avec les responsables au siège de la Fondation, mais aussi, plus directement, avec les représentants du groupe parlementaire CDU et la chancellerie fédérale. Il invita des représentants du PPE à Prague dans le but de légitimer ce partenaire jugé viable et proeuropéen ${ }^{(15)}$. La coalition anti-Mečiar fut présentée comme la seule voie permettant à la Slovaquie de rattraper le groupe des pays candidats avec lesquels les négociations d'adhésion allaient débuter en $1998^{(16)}$.

Sur ce terrain, comme sur d'autres, la Fondation Adenauer se trouva en concurrence avec les fondations américaines (notamment l'International Republican Institute) qui bénéficiaient de moyens financiers d'envergure et pouvaient financer directement les partis ${ }^{(17)}$. Cependant, c'est bien la KAS qui disposait de contacts dans les institutions communautaires chargées d'évaluer la situation dans le pays. À la différence

14 Cf. Laure Neumayer, L'enjeu européen dans les transformations postcommunistes, Paris, Belin, 2006.

15 Entretien avec un ancien représentant de la Fondation Konrad Adenauer, Berlin, 19 juillet 2000.

16 La Slovaquie n'avait pas été intégrée dans ce groupe, désigné lors du Conseil européen de Luxembourg en 1997, en raison des pratiques autoritaires du gouvernement Mečiar.

17 Cf. Geoffrey Pridham, "Complying with the European Union's Democratic Conditionality: Transnational Party Linkages and Regime Change in Slovakia, 1993-1998 ", Europe-Asia Studies, vol. 51, n 7, 1999, p. 1221-1244. 
des professionnels de la démocratisation américains, connus pour leur aide matérielle ponctuelle apportée aux campagnes électorales, les fondations allemandes proposaient un transfert de savoir-faire en matière électorale, voire une médiation auprès des fédérations européennes de partis. Au-delà du soutien matériel, c'est la présence sur le terrain, l'entretien de contacts à l'intérieur du champ politique, la familiarité avec les jeux politiques internes et la capacité à mobiliser les contacts internationaux qui déterminent la réception des fondations. Premier ministre de 1998 à 2006, Mikuláš Dzurinda entretint des liens étroits avec la Fondation Adenauer et le PPE. Élu au Parlement slovaque en 2012, il devint, l'année suivante, le président de la fondation proche du PPE (le Centre Martens).

Dans un autre contexte, le soutien aux formations et aux mouvements pro-européens pouvait s'avérer lourd de signification politique, comme en témoigne, plus récemment, le cas ukrainien. En Ukraine, les principales fondations allemandes se sont implantées à partir de la proclamation de l'indépendance du pays, en 1991. Si elles ont toutes cherché à travailler avec des partenaires modérés représentant des intérêts diversifiés (mouvements politiques, think tanks et centres de recherche, organisations syndicales), la dimension politique de ces activités pouvait varier. La Fondation Ebert évita de se rapprocher fortement de partis politiques et privilégiait une coopération avec les syndicats. En revanche, la Fondation Konrad Adenauer s'est démarquée par son appui octroyé à des mouvements politiques du centre et de droite, tout en proposant des programmes destinés à un spectre relativement large d'organisations politiques de jeunes ${ }^{(18)}$. Elle a apporté un soutien ouvert aux formations dirigées par Ioulia Timochenko et Victor Iouchtchenko au lendemain de la Révolution orange et a contribué à leur association au Parti populaire européen. Alors que les relations entre ces deux leaders devinrent de plus en plus conflictuelles et qu'ils ont été progressivement écartés du pouvoir, la Fondation Adenauer a soutenu, par l'intermédiaire de son représentant, la création, en 2010, du parti initié par Vitali Klitchko, l'Alliance démocratique ukrainienne pour la réforme (UDAR). Elle a proposé de nombreuses formations aux militants de ce mouvement, portant sur les aspects organisationnels, institutionnels et communicationnels du fonctionnement d'un parti politique.

Si la direction de la CDU a été régulièrement consultée et associée à ce travail d'ingénierie politique, ce dernier ne se résume pas à la médiation dans les relations partisanes bilatérales. En effet, c'est à un niveau européen plus multilatéral, celui des organisations partisanes regroupées autour du Parlement européen, qu'il faut chercher les interlocuteurs principaux de la Fondation Adenauer. C'est le Parti populaire européen qui suivait de près l'évolution de la situation politique en Ukraine par l'intermédiaire de son président Wilfried Martens ou encore de Jerzy Buzek lorsque ce dernier présidait le Parlement européen. De cette manière, le PPE recherchait, par l'intermédiaire de la KAS, des partenaires qui pouvaient témoigner de son assise dans les pays du voisinage européen. Cet intérêt pour les pays d'Europe du Centre-Est n'avait rien de nouveau: le PPE était déjà actif dans la région dans les années 1990 et la Fondation Adenauer

18 Ce faisant, la KAS coopère avec plusieurs think tanks spécialisés dans l'analyse politique tels que l'Institut de l'Éducation politique qu'elle a contribué à fonder, le Laboratoire des initiatives législatives, ainsi que l'Institut pour la coopération euro-atlantique. 
avait fourni une aide précieuse à l'association des formations politiques issues de la région, qui sont devenues, successivement, observatrices puis membres à part entière au moment des élargissements ${ }^{(19)}$.

Cela étant, l'intensification des violences politiques en Ukraine laisse à l'engagement pro-européen un goût amer. La mobilisation civique et politique autour de Maïdan Nézalejnosti (place de l'Indépendance) en 2013-2014, sa répression par les forces de l'ordre en février 2014 faisant une centaine de morts ont montré que l'engagement pro-européen était tout sauf une cause entendue. L'annexion de la Crimée par la Russie et la guerre fratricide qui ravage les régions de l'Est de l'Ukraine indiquent les limites du pouvoir des fondations allemandes. Si elles ont servi d'intermédiaire et fourni des contacts politiques aux factions qu'elles soutenaient en temps de paix, elles ne sont pas en mesure d'apporter le soutien que réclament les forces pro-européennes au pouvoir à Kiev. Dans cette situation, il ne reste aux fondations, après avoir attisé la flamme pro-européenne auprès des militants politiques, que le repli sur leurs affaires quotidiennes de la formation des syndicalistes ou d'organisation des séminaires sur les questions économiques, climatiques ou celles relatives au passé. Le dialogue sur la mise en œuvre de l'accord d'association UE-Ukraine ratifié en septembre 2014 ouvre cependant une voie à la continuation du dialogue sur les enjeux européens.

Le travail des fondations politiques allemandes est indissociable de leur engagement européen, marqué par leur présence physique à Bruxelles et par leur soutien aux élites pro-européennes dans les pays partenaires. S'il pouvait paraître comme un enjeu consensuel dans certains moments du processus de pré-adhésion, l'investissement pro-européen impliquait des choix qui pouvaient contribuer à accentuer des clivages, voire à attiser les tensions entre les formations et personnalités soutenues par les fondations et celles qui ne rentraient pas dans ce cadre. Si l'engagement pro-européen peut être un facteur de reconnaissance en Allemagne et dans les pays gouvernés par des forces favorables à l'intégration européenne, dans d'autres contextes il peut prendre une connotation plus clivante. Il n'empêche, le dispositif des fondations permet à la chancellerie fédérale de suivre, en direct, les évolutions politiques dans le voisinage européen et de garder, ainsi, tous les canaux ouverts.

\begin{abstract}
The work of the German political foundations has an important European dimension due to their presence in Brussels and to their support to pro-European elites in the partner countries. While this European investment is usually presented as a consensual issue, this contribution sheds light on the tensions and paradoxes that it may cause in some configurations. The article analyses the foundations' investment in transnational party networks and their implication in regime changes in Europe. The Ukrainian case is used to show the limits of the political foundations' action.
\end{abstract}

